\title{
Gap junctions in C. elegans
}

\author{
Karina T. Simonsen ${ }^{1}$, Donald G. Moerman ${ }^{2}$ and Christian C. Naus ${ }^{1 *}$ \\ ${ }^{1}$ Department of Cellular and Physiological Sciences, Life Sciences Institute, University of British Columbia, Vancouver, BC, Canada \\ 2 Department of Zoology and Michael Smith Laboratories, University of British Columbia, Vancouver, BC, Canada
}

\section{Edited by:}

Georg Zoidl, York University, Canada

Reviewed by:

Alberto Passi, Università

dell'Insubria, Italy

Citlali Trueta, Instituto Nacional de

Psiquiatria Ramon de la Fuente

Muñiz, Mexico

${ }^{*}$ Correspondence:

Christian C. Naus, Department of

Cellular and Physiological Sciences,

Life Sciences Institute, University of

British Columbia, 2350 Health

Sciences Mall, Vancouver, BC V6R

1W9, Canada

e-mail:christian.naus@ubc.ca
As in other multicellular organisms, the nematode Caenorhabditis elegans uses gap junctions to provide direct cell-to-cell contact. The nematode gap junctions are formed by innexins (invertebrate analogs of the connexins); a family of proteins that surprisingly share no primary sequence homology, but do share structural and functional similarity with connexins. The model organism $C$. elegans contains 25 innexin genes and innexins are found in virtually all cell types and tissues. Additionally, many innexins have dynamic expression patterns during development, and several innexins are essential genes in the nematode. $C$. elegans is a popular invertebrate model due to several features including a simple anatomy, a complete cell lineage, sequenced genome and an array of genetic resources. Thus, the worm has potential to offer valuable insights into the various functions of gap junction mediated intercellular communication.

\section{Keywords: $C$. elegans, gap junctions, innexins, intercellular communication}

\section{GAP JUNCTIONS AND INNEXINS}

Members of the innexin gene family (innexins: invertebrate analogs of connexins) in C. elegans were first described in 1996 (Starich, 1996), and with the complete sequence of the C. elegans genome it was revealed that the family consists of 25 genes (C. elegans sequencing Consortium, 1998). The C. elegans genome, as well as all other invertebrate genomes, contains no connexin homologs, and the innexins share no sequence homology with the connexins. Based on sequence homology with the innexins, a third gene family was identified in vertebrates and named pannexins. Innexins share a similar topology with both connexins and pannexins. This consists of four transmembrane helices, two extracellular loops, and $\mathrm{N}$ and $\mathrm{C}$ termini located on the cytoplasmic side. Two cysteine residues in each of the predicted extracellular loops are conserved among all innexins. The two extracellular loops of innexins are thought to be longer than in the connexins, i.e., $\sim 50$ amino acids for innexins and $\sim 30$ amino acids for connexins (Phelan, 2005; Oshima et al., 2013). Further differences were noted when C. elegans INX-6 was examined structurally and the molecular dimensions of the junction formed by INX-6 was observed to be greater than those of Cx26; this may be due to greater spacing between adjacent membranes (Oshima et al., 2013).

Gap junctions form when two hemichannels from adjacent cells line up to make a channel; in vertebrates the hemichannels are hexamers of connexin or pannexin proteins and in invertebrates the innexins are also believed to form hexameric channels (Oshima et al., 2013). If the two hemichannels are identical, the gap junction is referred to as homotypic; if the two hemichannels differ, the gap junction is heterotypic. If the hemichannels are composed of only one type of connexin, pannexin or innexin they are called homomeric, and if more than one type of protein is present within the channel, then the gap junction is called heteromeric. In C. elegans homotypic gap junctions have been suggested for the majority of innexins studied, namely INX-3,
EAT-5, INX-6, INX-16 and INX-19 while UNC-7 and UNC-9 seem to be able to form both homotypic and heterotypic gap junctions. INX-14 and INX-22 are suggested to form heteromeric gap junctions, and these two innexins or perhaps only INX-14 is further suggested to form heterotypic gap junctions with INX-8 and/or INX-9. INX-19 is the only innexin for which a potential function as a hemichannel has been reported (see below for details).

Null and missense mutations are available for all $25 \mathrm{mem}$ bers of the gene family and a range of phenotypes have been reported, including lethality for at least five of the mutant genes, demonstrating the functional importance of gap junctions in the nematode. Unfortunately, detailed functional studies of the C. elegans innexins are available for less than half of this protein family.

There are 25 C. elegans innexins contributing to gap junctions that are present in virtually all tissue and cell types in the nematode (see Table 1). A comprehensive expression map of all innexins provides potential insights into function (Altun et al., 2009). Promoter regions of all innexins were fused individually to Green Fluorescent Protein (GFP) and visualized throughout the lifespan of the nematodes and a complex and dynamic picture of the expression of innexin genes emerged from this study. Some innexins are expressed mostly during embryogenesis, others mainly in the adult animals and some are constitutively expressed throughout the entire lifespan. Most innexin genes are expressed in multiple tissues, but a few were found to be expressed in only one cell type. This study provides a large amount of detail on innexin expression; however, due to the nature of the experiments using only promoter regions and not full-length protein fusions, not all innexin expression is represented. For example, the authors note that in some cells previously shown to make gap junctions by electron microscopy, no expression of innexins was observed in their study (Altun et al., 2009). 
Table 1 | C. elegans innexins.

\begin{tabular}{|c|c|c|c|c|}
\hline Gene & Sequence & $\begin{array}{l}\text { Protein } \\
\text { size (aa) }\end{array}$ & Expression & Phenotype (mutant) \\
\hline in $x-1$ & C16E9.4 & 428 & Neurons, muscle & - \\
\hline in $x-2$ & F08G12.10 & 419 & Pharynx & - \\
\hline in $x-3$ & F22F4.2 & 420 & Pharynx, neurons & Lethal \\
\hline in $x-5$ & R09F10.4 & 447 & Hypodermis, seam cells, vulval cells & - \\
\hline in $x-6$ & $\mathrm{C} 36 \mathrm{H} 8.2$ & 389 & Pharynx & Asynchronous pumping in pharynx \\
\hline in $x-7$ & K02B2.4 & 556 & Pharynx, neurons & Reduced brood size \\
\hline in $x-8$ & ZK792.2 & 382 & Gonadal sheath cells & - \\
\hline in $x-9$ & ZK792.3 & 382 & Gonadal sheath cells & - \\
\hline $\operatorname{in} x-10$ & $\mathrm{~T} 18 \mathrm{H} 9.5$ & 559 & Pharynx, gonadal sheath cells, neurons, vulval cells, muscles & - \\
\hline $\operatorname{in} x-11$ & W04D2.3 & 529 & Pharynx, neurons, intestine, vulval cells, muscles & - \\
\hline in $x-12$ & ZK770.3 & 408 & Excretory cell, neurons, glial-like sheath and socket cells, vulval cells & Lethal \\
\hline in $x-13$ & Y8G1A.2 & 385 & Excretory cell, neurons, glial-like sheath and socket cells, vulval cells & Lethal \\
\hline in $x-14$ & F07A5.1 & 432 & Germ cells & Lethal \\
\hline in $x-15$ & R12E2.9 & 382 & Intestine & - \\
\hline inx-16 & R12E2.5 & 372 & Intestine & Reduced brood size, constipated \\
\hline in $x-17$ & $\mathrm{R} 12 \mathrm{E} 2.4$ & 362 & Intestinal-rectal valve & - \\
\hline in $x-18$ & $\mathrm{C} 18 \mathrm{H} 7.2$ & 436 & Neurons, muscles & - \\
\hline inx-19 (or nsy-5) & $\mathrm{T} 16 \mathrm{H} 5.1$ & 454 & Neurons & Chemotaxis defective \\
\hline $\operatorname{in} x-20$ & $\mathrm{~T} 23 \mathrm{H} 4.1$ & 483 & Pharynx, intestinal-rectal valve & - \\
\hline inx-21 & Y47G6A. 1 & 481 & Intestine & - \\
\hline $\operatorname{in} x-22$ & Y47G6A.2 & 462 & Germ cells & Lethal \\
\hline eat-5 & F13G3.8 & 423 & Pharynx & Asynchronous pumping in pharynx \\
\hline che-7 (or in $x-4$ ) & F26D11.10 & 554 & Neurons & Chemotaxis defective \\
\hline unc-7 & R07D5.1 & 522 & Neurons, muscles & Uncoordinated movement \\
\hline unc-9 & $\mathrm{R} 12 \mathrm{H} 7.1$ & 386 & Neurons, muscles & Uncoordinated movement \\
\hline
\end{tabular}

Table made using information from Wormbase release 220 (www.wormbase.org) and papers cited within the text.

All C. elegans innexins have orthologs in the sequenced Caenorhabditis species C. briggsae and C. remanei (except inx-8 and in $x-9$ which share a single ortholog in C. briggsae), suggesting that all 25 innexins are true genes, not pseudogenes (Altun et al., 2009). Three pairs of the innexins are polycistronic: inx-12 and inx-13, inx-16 and inx-17, and inx-21 and inx-22. In C. elegans, $15 \%$ of genes are found in operons, but many operons have been shown to be hybrid operons, meaning that the genes can be co-transcribed as well as being individually transcribed (Blumenthal, 2005). The innexin pairs seem to be expressed in a similar manner; however, they do not seem to give rise to identical phenotypes. For example, RNAi of inx-16 but not inx-17 causes a constipated phenotype and inx-22 but not inx-21 was identified in screens for repressors of oocyte maturation (Govindan et al., 2006; Peters et al., 2007; Whitten and Miller, 2007). Thus, based on current knowledge for these innexins, it seems likely that they are part of hybrid operons, but the functional importance of this observation is yet to be determined.

Gap junctions permit electrical coupling between cells, as well as passage of small molecules that initiate signal transduction and gene expression. More recently, channel-independent functions have also been proposed (Vinken et al., 2012). In C. elegans, electron microscopy has detected gap junctions between neurons with axon-to-axon and axon-to-soma contacts being common and soma-to-soma contacts being less common. Around $10 \%$ of the synapses in the C. elegans nervous system are comprised by gap junctions and obviously, they are vital for the animal (White et al., 1986). Gap junctions are also present between socket and sheath cells (glial cells), but not between glia and neurons, and between gonadal sheath cells and between oocytes and sheath cells, but not between germ cells and sheath cells in the distal gonad (Hall et al., 1999, 2008). Both the pharyngeal muscles and the body wall muscles in C. elegans are connected by gap junctions. Electrical coupling between body wall muscles is what allows for the coordination in the sinusoidal movement of nematodes. The intestinal cells also form gap junctions with one another and the hypodermis makes several gap junctions to neighboring tissues, including seam cells and the excretory canal (Hall et al., 2008; Peters et al., 2007; Altun et al., 2009). Clearly, gap junctions are important in C. elegans; however, the specific innexins comprising the gap junctions are far from fully understood. Below we summarize what is known about specific innexin genes.

\section{C. elegans INNEXINS}

\section{INX-3}

INX-3 was the first C. elegans innexin shown to form an intercellular channel. Expression of INX-3 in Xenopus oocytes enabled electrical coupling between paired oocytes. Notably, the gating properties were indistinguishable from those of connexins (Landesman et al., 1999). Light microscopy shows INX-3 at 
plaque-like structures at cell-cell boundaries, as expected for gap junctions, and electron microscopy confirms that INX-3 is indeed part of gap junctions in C. elegans. INX-3 is expressed throughout embryonic development and the protein is essential for survival in C. elegans. Most inx-3 mutants die during embryogenesis and the few larvae that hatch never make it past the L1 stage (see Box 1). The hatched larvae have short or sometimes a detached pharynx and the pumping in pharynx is unsynchronized, like mutants lacking eat-5 and inx-6 (see below). In the adult worm, the INX-3 protein can only be detected in the posterior pharynx. Overexpression of in $x-3$ causes terminal defects during embryogenesis similar to the ones observed in the in $x-3$ mutant, so obviously this innexin is crucial for this developmental process, but the exact function remains unknown (Starich et al., 2003).

\section{EAT-5 AND INX-6}

eat-5 mutants were named based on their abnormal eating; they display unsynchronized muscle contractions in the pharynx, the organ responsible for capturing bacterial food, grinding it up and transporting it to the intestine (see Box 1) (Starich, 1996). The pharyngeal muscles are electrically coupled by gap junctions, much like cardiac muscle cells, and in eat-5 mutants, injected carboxyfluorescein fails to spread into adjacent muscle cells of the pharynx (Starich, 1996), suggesting that the gap junctions are formed by EAT-5. An attempt to induce electrical coupling in Xenopus oocytes with eat-5 failed, however, and no coupling could be detected even when eat-5 was co-expressed with inx3 , an innexin with partially overlapping expression with eat-5 (Landesman et al., 1999). This does not rule out that EAT-5 forms genuine gap junctions, since the observed lack of coupling could be due to lack of the right innexin partner being present, or the channel formed may not be active due to lack of post-translational modification. Inability to couple Xenopus oocytes has also been observed for a few connexins e.g., connexin33 and connexin31.1 (Landesman et al., 1999).

in $x-6$ is another innexin expressed in the pharyngeal muscles in a similar pattern to eat-5, and, as for eat-5, synchronized muscle contractions in the pharynx is lost in this mutant ( $\mathrm{Li}$ et al., 2003). The inx-6 mutant animals also display similar defects in dye spreading as that observed for the eat -5 mutant, and interestingly, EAT- 5 could partially substitute for INX-6 function, hinting at very similar, though not identical, roles for these two innexins. in $x-6$ is a cold-sensitive mutant and at the restrictive temperature it arrests development after hatching. This strong phenotype is due to a point mutation in a non-conserved area in the $\mathrm{C}$ terminus of the protein, suggesting this intracellular region is crucial for correct protein function.

Recently, recombinant INX-6 channels were purified and electron microscopy revealed loosely packed hexagonal gap junction plaques with a larger channel diameter than that observed

\section{Box 1 | C. elegans-an excellent model organism.}

C. elegans is a small $(\sim 1 \mathrm{~mm})$, non-parasitic, transparent nematode with a fairly simple anatomy and the adult animal has only 959 somatic cells. It is easily cultured in the laboratory on agar plates seeded with $E$. coil bacteria and it grows from embryo to fertile adult in approximately 3 days at $20^{\circ} \mathrm{C}$. The worm goes through four larval stages, L1-L4, and then reaches adulthood. Limitations in food or other stress factors at the L1-L2 stage causes the larvae to enter dauer stage, a stress-resistant, and long-lived diapause stage. When food becomes available, the dauer will resume development. The total lifespan of $C$. elegans is around 3 weeks.

The animal is hermaphroditic and self-fertilizing, as it produces both oocytes and sperm. Hermaphrodites have two X chromosomes, while males have a single $X$ chromosome. Male and hermaphrodite crosses can be done for genetic studies. Adult hermaphrodites, when selffertilizing, will produce around 300 progeny. Mutant animals can be obtained by chemical mutagenesis and strains can be stored frozen for extended periods of time (decades). Many mutants can be maintained as self-fertilizing hermaphrodites on plates. Transgenic animals, for example animals expressing a GFP fusion protein, can be generated by microinjection or bombardment of exogenous DNA. RNA-mediated interference (RNAi) is readily obtained in $C$. elegans by feeding worms $E$. coil expressing dsRNA corresponding to the gene of interest. $40 \%$ of $C$. elegans genes have human orthologs (greater than 7000 genes).

C. elegans feeds by pumping bacteria into the pharynx, a tube-like muscular pump with its own nervous system. Bacteria trapped in the pharynx are broken up by muscle contractions and passed into the intestine, the digestive organ extending almost the entire length of the animal. The nervous system of the worm is comprised of 302 neurons and the complete wiring is known. Most neurons are located in the head of the worm, and cells of the nervous system are organized into ganglia in the head and tail. Body wall muscles run along the whole length of the animal in four quadrants and smaller muscles are located in the pharynx and around the intestine and the vulva. The reproductive system in the hermaphrodite consists of two gonadal arms joined by a shared uterus. Germ cells in the distal part of the gonad are undifferentiated and mature as they move proximally. The mature oocytes are ovulated into the sperm-containing spermatheca, where they are fertilized before moving into the uterus. Figure adapted from www.wormatlas.org.

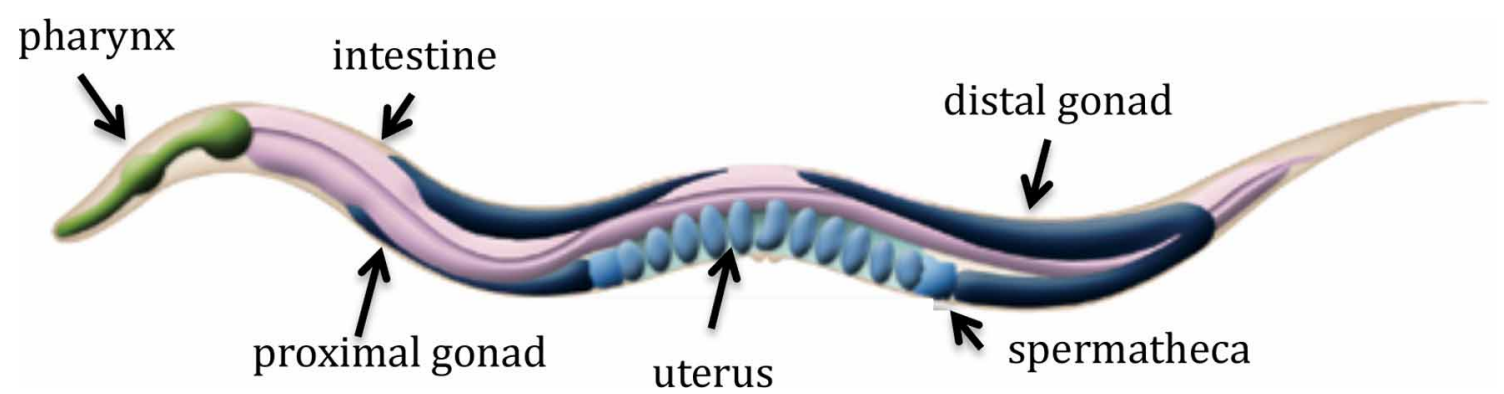


for connexins $(\sim 140 \AA$ for INX-6 as compared with $92 \AA$ for connexin26). Microinjection of low molecular weight fluorescent dyes indicated that the INX- 6 channel is more permeable than connexin 43 , as the INX-6 channels allowed passage of $3 \mathrm{kDa}$ and even some $10 \mathrm{kDa}$ tracers. Curiously, INX-6 formed gap junctions when expressed in the insect cell line Sf9 but did not form gap junctions in mammalian HeLa cells, which could reflect the need of a modifier or modification for INX-6 to form actual channels (Oshima et al., 2013).

\section{INX-16}

The innexin gene inx-16 is expressed in the intestine and the protein localizes to cell-cell contacts between intestinal cells, consistent with this innexin being a gap junction protein. Observations in a mosaic worm (in which some cells are wild type and some cells are mutant) suggest that INX-16 forms homotypic channels, since the protein is absent from cell-cell contacts between inx-16 expressing and non-expressing intestinal cells (Peters et al., 2007). The mutant is constipated and, presumably as a result of this, small, slow growing and gives rise to fewer progeny than wild type C. elegans. INX-16 allows $\mathrm{Ca}^{2+}$ waves to travel between intestinal cells and since this calcium flux is required for intestinal muscle contractions, the mutant's defecation cycle is disrupted, leaving the animal constipated. The absence of $\mathrm{Ca}^{2+}$ flux in the intestine was also observed in a different study where the mutant blocked the spread of an endogenous fluorescent marker in the intestine, a phenomenon shown to be preceded by a $\mathrm{Ca}^{2+}$ wave (Coburn et al., 2013). Dye transfer of Lucifer yellow between the intestinal cells was not inhibited in the inx-16 mutant (Peters et al., 2007), but other innexins are also likely to be expressed in the intestine, including inx-2, inx-11 and inx-15 (Altun et al., 2009). Despite the potential for several gap junctions to function in the intestine, INX-16 is clearly necessary for allowing $\mathrm{Ca}^{2+}$ waves to propagate throughout the intestine.

\section{INX-22 AND INX-14}

The adult hermaphrodite gonad consists of two U-shaped arms that share a common uterus; the germ cells in the distal gonad are undifferentiated and as they move to the proximal gonad (closest to the uterus) they mature and the oocytes will move into the sperm-containing spermatheca, where they are fertilized (see Box 1). Subsequently they move into the uterus and ultimately, the eggs are laid through the vulva. Gonadal sheath cells (myoepithelial cells) cover the proximal gonad arm and most of the distal gonad arm; they form gap junctions with each other and with the proximal germline, as has been shown by electron microscopy (Hall et al., 2008). INX-14 and INX-22 are expressed in the germ cells and co-localize in plaque-like structures at the interface between sheath cells and oocytes in the proximal and distal gonad, consistent with these two innexins forming gap junctions (Govindan et al., 2009). In an inx-22 null mutant, immunostaining showed that INX-14 was absent from the proximal gonad, but interestingly, INX-14 localized almost normally in the distal gonad (Edmonds et al., 2011). One interpretation of these results is that in the proximal gonad, INX-14 and INX-22 form heteromeric gap junctions. Null mutation in inx-14 causes sterility due to defects in oogenesis (Govindan et al., 2009), but null mutation in inx-22 does not show this phenotype; these mutants are fertile, although they have reduced brood size. This is the expected result if these proteins act together in the proximal gonad but redundantly in the distal gonad.

These two innexins were both identified as repressors of oocyte maturation in the absence of sperm [in a fog-2(q71) background, which generates XX females and XO males] (Govindan et al., 2006; Whitten and Miller, 2007). In C. elegans the process of meiotic maturation is tightly coupled to sperm availability with sperm secreting the signaling molecule major sperm protein (MSP), which acts as a paracrine hormone and induces oocyte maturation and sheath contraction. The extracellular MSP gradient in the gonad of hermaphrodites is not believed to be high enough to reach the loop region of the gonad where stimulation of oocyte maturation takes place, and the sheath cells are hypothesized to communicate the presence of the MSP signal throughout the whole germline since the sheath cells are coupled to one another and to the germline via gap junctions. The two screens identifying inx-14 and inx-22 as negative regulators of oocyte maturation were both RNAi screens and would not have picked up any innexins with redundant functions (Whitten and Miller, 2007).

Further functional studies highlight differences between INX14 and INX-22; INX-14 is required to inhibit oocyte maturation only in the absence of sperm, INX-22 is required in the presence and absence of sperm (Whitten and Miller, 2007). INX-14, but not INX-22, was also shown to be important in the process of guiding male sperm, deposited during mating, from the vulva to the spermatheca, where fertilization takes place. Absence of INX14 causes defects in velocity and directionality of sperm migration (Edmonds et al., 2011). Also in this process, INX-14 was shown to function within the germ cells, so in order to identify potential innexins partner(s) in the sheath cells the four innexins shown to be expressed in the gonadal sheath cells (inx-5, inx-8, inx-9, and inx-10) (Altun et al., 2009) were tested, and RNAi of inx-8 and in $x-9$ showed similar sperm guidance defects. As these genes are $85 \%$ identical, RNAi of either gene is predicted to target both genes, so the results for these two innexins cannot be separated. Oocytes secrete prostaglandins to guide sperm to the spermatheca and signaling via innexins in gap junctions is suggested to control prostaglandin transport and/or activity (Edmonds et al., 2011).

Taken together, signals from the gonadal sheath cells to the germ cells seem to be communicated via gap junctions made of the innexins INX-14 and INX-22 and most likely INX-8 and INX-9, with the possibility of even more innexins being involved. Interestingly, the gene inx-14 was also identified in a forward genetic screen for mutants resistant to the pathogenic bacteria $S$. aureus. Subsequently, in $x-8$ and inx- 9 were also tested and they too showed resistance (Miyata et al., 2008).

\section{UNC-7 AND UNC-9}

Mutants of the genes unc-7, the first innexin identified in C. elegans, and unc-9 display identical uncoordinated movement phenotypes often referred to as kinking: the animals move in very severe bending motions instead of the normal sinusoidal body bends as generated in wild type nematodes. UNC-7 and UNC-9 are both expressed throughout the nervous system as well as in body muscles. There is extensive co-localization of these two proteins in the nervous system, but in body wall muscles UNC-9 
is reported to be expressed at a higher level than UNC-7. These expression data used an unc-9::gfp construct which only partially rescued the uncoordinated phenotype, maybe due to GFP interfering with UNC-9 function (Starich et al., 2009). Both innexins are able to electrically couple paired Xenopus oocytes and they both form homotypic and heterotypic gap junctions, but no evidence for heteromeric gap junctions was found (Starich et al., 2009). Since removal of functional UNC-7 or UNC-9 in muscles does not lead to an uncoordinated phenotype, it is suggested that these two innexins are required in the nervous system for coordinated locomotion.

Despite the similar phenotypes of mutants in $u n c-7$ and $u n c-9$ and similar expression patterns of the two genes, functional studies show large differences between the two innexins. Body wall muscles in $C$. elegans are electrically coupled via gap junctions and this coupling is significantly reduced in the unc- 9 mutants, but not in the unc-7 mutant (Liu, 2006). Remarkably, five other innexins have been shown to also contribute to the electrical coupling in the body wall muscles: INX-1, INX-10, INX-11, INX-16, and INX-18 (Liu et al., 2013). The stomatin-like protein UNC-1 is also involved in this electrical coupling of the body wall muscles in C. elegans, and UNC-1 co-localizes with UNC-9 at intercellular junctions in body wall muscles. Bimolecular fluorescence complementation assays confirmed that these two proteins are in very close proximity (Chen et al., 2007). Localization of UNC-9 is not dependent on UNC-1, nor is UNC-1 dependent on UNC-9 for guidance or localization. This has led these authors to speculate that the primary function of UNC-1 may be to modulate the gating of gap junctions. This is the only study in C. elegans to possibly identify an interacting partner for one of the innexins. UNC-9 and $\mathrm{UNC}-1$ are both required for synchronized action potentials and $\mathrm{Ca}^{2+}$ transients between neighboring body wall muscle cells, demonstrating that UNC-9 gap junctions together with UNC-1 are involved in synchronizing muscle activities (Liu et al., 2011).

\section{INX-19 (NSY-5)}

The gap junction protein INX-19 (also known as NSY-5, for neuronal symmetry) is expressed in a subset of neurons in the head and tail and was identified in a genetic screen for mutants lacking asymmetrical AWC olfactory neurons. The pair of AWC neurons are morphologically symmetric, but they differ in their ability to sense different chemicals due to different genes being expressed in either the left or right neuron (Chuang et al., 2007). INX-19 can electrically couple Xenopus oocytes and measurements in the Xenopus system also showed a hemichannel action for this innexin, thus INX-19 may be able to form gap junctions and perhaps even functional hemichannels (Chuang et al., 2007). Expression was observed during embryogenesis, with the highest levels of INX-19 in late embryogenesis and at the L1 larval stage; in the adult worm only weak expression was observed in several neurons excluding the AWC olfactory neuron pair. Serial-section electron microscopy on embryos and L1 larvae revealed extensive gap junction coupling between cell bodies of the AWC neurons and other neurons, and in mutants these gap junctions are missing. The gap junctions are not present in adult worms (Chuang et al., 2007). Dye transfer assays using a caged photoactivatable fluorescent dye in isolated embryonic neurons containing either a wild-type inx-19 gene or an inx-19 loss-of function mutation demonstrated that INX-19 is crucial in these neurons for dye transfer (Schumacher et al., 2012). Collectively, this is strong evidence that INX-19 forms functional gap junctions allowing for the passage of small molecules between neurons in the developing nematode. The sub-cellular location of this innexin was further studied by expressing in $x-19$ fused to GFP in COS cells (fibroblast-like cell line derived from monkeys), showing that INX-19 localized to plaques at cell-cell contacts when both cells were expressing this innexin, suggesting the formation of a homotypic gap junction (Chuang et al., 2007).

In wild type animals, one $\mathrm{AWC}^{\mathrm{ON}}$ and one $\mathrm{AWC}^{\mathrm{OFF}}$ neuron are always generated, where the $\mathrm{ON}$ and $\mathrm{OFF}$ denotes the expression of the reporter str-2::gfp. Removal of inx-19 caused two AWC ${ }^{\text {OFF }}$ neurons, whereas overexpression of inx-19 generated two $\mathrm{AWC}^{\mathrm{ON}}$ neurons, clearly showing the importance of this innexin in establishing the asymmetry of the AWC neurons. By expressing vertebrate $\mathrm{Ca}^{2+}$-buffer proteins within the INX-19 network, the AWC asymmetry was disrupted, thereby indicating that $\mathrm{Ca}^{2+}$ signaling is involved in establishing the left-right asymmetry in C. elegans AWC olfactory neurons via an INX-19 transient gap junctional network (Schumacher et al., 2012).

\section{POTENTIAL OF C. elegans AS A MODEL FOR THE STUDY OF GAP JUNCTIONS}

C. elegans has 25 innexins and, so far, in-depth functional analysis has been done for less than half of the genes including inx-3, inx-6, eat-5, inx-16, inx-14, inx-22, inx -19 , unc-7, and unc-9. All these innexins seem to act as genuine gap junctions, as evidenced by a variety of techniques; electron microscopy, Xenopus oocyte coupling, expression of innexins in COS cells and Sf9 cells, fluorescent dye transfer assays, studies of GFP fusion-proteins, voltage clamp and RNAi. One of the most interesting results to come from these studies was the identification of a modifier of gap junctions formed by UNC-9, namely the stomatin-like protein UNC-1 (Chen et al., 2007). There has been substantial work done in identifying connexin interacting proteins/modifiers (Laird, 2010), and this area may hold many more exciting findings for C. elegans innexins. Another important finding is that channels formed by C. elegans INX-6 show a more permeable gap junction with a larger channel diameter than has been reported for connexins (Oshima et al., 2013). The implications of this observation are not clear at this time. Several interesting observations pertaining to the role of innexins in the biology of C. elegans have emerged from these studies including roles in movement, fertilization, oocyte maturation, cell identity and innate immunity. In other words, innexins are involved in almost all aspects of the development of this organism.

When studying gap junctions, dye transfer assays and specific inhibitors are often used, and in C. elegans these approaches have been used in various cell types. When carboxyfluorescein was injected into muscle cells of dissected pharynxes, it spread to the remainder of pharyngeal muscles in wild type worms, but not in eat-5 mutant animals (Starich, 1996). Another fluorescent dye, Lucifer yellow, was injected into the intestinal cells of C. elegans and here the dye transferred throughout the intestine (Peters et al., 2007). Carboxyfluorescein was also injected into body wall muscle cells, but here the dye did not diffuse into neighboring cells (Liu, 2006). Isolated embryonic neurons were cultured and 
loaded with an photoactivatable (or caged) fluorescent dye, which after uncaging by UV light travelled to neighboring cells through gap junctions made of INX-19 (Schumacher et al., 2012). The gap junction blocker carbenoxolone and the pannexin blocker probenecid were tested on UNC-9 in body wall muscles, but none of these chemical inhibitors showed any effect (Liu et al., 2011).

Although the vertebrate connexins and invertebrate innexins are completely unrelated by sequence, they are both the molecular building blocks of gap junctions and thus enable intercellular communication. Much is still unknown for innexins-Are they post-translationally modified? Can they form functional hemichannels? Do any of them have channel-independent functions? Perhaps the biggest question still to be answered is, what small molecules are transported through the innexin gap junctions? C. elegans is a small animal model with advantages for studying cell-cell communication, and this nematode has the potential to contribute a great deal to our understanding of how gap junctions function.

\section{ACKNOWLEDGMENTS}

Karina T. Simonsen is supported by The Danish Council for Independent Research, Natural Sciences (FNU). Donald G. Moerman is a Senior Fellow of the Canadian Institute For Advanced Research. Christian C. Naus holds a Canada Research Chair and is a Member of the Canadian Academy of Health Sciences.

\section{REFERENCES}

Altun, Z. F., Chen, B., Wang, Z.-W., and Hall, D. H. (2009). High resolution map of Caenorhabditis elegansgap junction proteins. Dev. Dyn. 238, 1936-1950. doi: 10.1002/dvdy.22025

Blumenthal, T. (2005). “Trans-splicing and operons," in WormBook, ed The C. elegans Research Community, WormBook. doi: 10.1895/wormbook.1.5.1

Chen, B., Liu, Q., Ge, Q., Xie, J., and Wang, Z.-W. (2007). UNC-1 regulates gap junctions important to locomotion in C. elegans. Curr. Biol. 17, 1334-1339. doi: 10.1016/j.cub.2007.06.060

Chuang, C.-F., VanHoven, M. K., Fetter, R. D., Verselis, V. K., and Bargmann, C. I. (2007). An innexin-dependent cell network establishes left-right neuronal asymmetry in C. elegans. Cell 129, 787-799. doi: 10.1016/j.cell.2007.02.052

Coburn, C., Allman, E., Mahanti, P., Benedetto, A., Cabreiro, F., Pincus, Z., et al. (2013). Anthranilate fluorescence marks a calcium-propagated necrotic wave that promotes organismal death in C. elegans. PLoS Biol 11:e1001613. doi: 10.1371/journal.pbio. 1001613

Consortium, T. C. E. S. (1998). Genome sequence of the nematode C. elegans: a platform for investigating biology. Science 282, 2012-2018. doi: 10.1126/science.282.5396.2012

Edmonds, J. W., McKinney, S. L., Prasain, J. K., and Miller, M. A. (2011). The gap junctional protein INX-14 functions in oocyte precursors to promote C. elegans sperm guidance. Dev. Biol. 359, 47-58. doi: 10.1016/j.ydbio.2011.08.014

Govindan, J. A., Cheng, H., Harris, J. E., and Greenstein, D. (2006). Gao/i and Gas signaling function in parallel with the MSP/Eph receptor to control meiotic diapause in C. elegans. Curr. Biol. 16, 1257-1268. doi: 10.1016/j.cub.2006.05.020

Govindan, J. A., Nadarajan, S., Kim, S., Starich, T. A., and Greenstein, D. (2009). Somatic cAMP signaling regulates MSP-dependent oocyte growth and meiotic maturation in C. elegans. Development 136, 2211-2221. doi: 10.1242/dev.034595

Hall, D. H., and Altun, Z. F. (2008). C. elegans Atlas. New York, NY: Cold Spring Harbor Laboratory Press.

Hall, D. H., Winfrey, V. P., Blaeuer, G., Hoffman, L. H., Furuta, T., Rose, K. L., et al. (1999). Ultrastructural features of the adult hermaphrodite gonad of Caenorhabditis elegans: relations between the germ line and soma. Dev. Biol. 212, 101-123. doi: 10.1006/dbio.1999.9356

Laird, D. W. (2010). The gap junction proteome and its relationship to disease. Trends Cell Biol. 20, 92-101. doi: 10.1016/j.tcb.2009.11.001

Landesman, Y., White, T. W., Starich, T. A., Shaw, J. E., Goodenough, D. A., and Paul, D. L. (1999). Innexin-3 forms connexin-like intercellular channels. J. Cell Sci. 112, 2391-2396.
Li, S., Dent, J. A., and Roy, R. (2003). Regulation of intermuscular electrical coupling by the Caenorhabditis elegans innexin inx-6. Mol. Biol. Cell 14, 2630-2644. doi: 10.1091/mbc.E02-11-0716

Liu, P., Chen, B., Altun, Z. F., Gross, M. J., Shan, A., Schuman, B., et al. (2013). Six innexins contribute to electrical coupling of $C$. elegans body-wall muscle. PLoS ONE 8:e76877. doi: 10.1371/journal.pone.0076877

Liu, P., Chen, B., and Wang, Z. W. (2011). Gap junctions synchronize action potentials and $\mathrm{Ca} 2+$ transients in Caenorhabditis elegans body wall muscle. J. Biol. Chem. 286, 44285-44293. doi: 10.1074/jbc. M111.292078

Liu, Q. (2006). Low conductance gap junctions mediate specific electrical coupling in body-wall muscle cells of Caenorhabditis elegans. J. Biol. Chem. 281, 7881-7889. doi: 10.1074/jbc.M512382200

Miyata, S., Begun, J., Troemel, E. R., and Ausubel, F. M. (2008). DAF-16dependent suppression of immunity during reproduction in Caenorhabditis elegans. Genetics 178, 903-918. doi: 10.1534/genetics.107.083923

Oshima, A., Matsuzawa, T., Nishikawa, K., and Fujiyoshi, Y. (2013). Oligomeric structure and functional characterization of Caenorhabditis elegans innexin-6 gap junction protein. J. Biol. Chem. 288, 10513-10521. doi: 10.1074/jbc.M112.428383

Peters, M. A., Teramoto, T., White, J. Q., Iwasaki, K., and Jorgensen, E. M. (2007). A calcium wave mediated by gap junctions coordinates a rhythmic behavior in C. elegans. Curr. Biol. 17, 1601-1608. doi: 10.1016/j.cub.2007. 08.031

Phelan, P. (2005). Innexins: members of an evolutionarily conserved family of gap-junction proteins. Biochim. Biophys. Acta 1711, 225-245. doi: 10.1016/j.bbamem.2004.10.004

Schumacher, J. A., Hsieh, Y.-W., Chen, S., Pirri, J. K., Alkema, M. J., Li, W.-H., et al. (2012). Intercellular calcium signaling in a gap junction-coupled cell network establishes asymmetric neuronal fates in C. elegans. Development 139, 4191-4201. doi: 10.1242/dev.083428

Starich, T. A. (1996). eat-5 and unc-7 represent a multigene family in Caenorhabditis elegans involved in cell-cell coupling. J. Cell Biol. 134, 537-548. doi: $10.1083 /$ jcb.134.2.537

Starich, T. A., Miller, A., Nguyen, R. L., Hall, D. H., and Shaw, J. E. (2003). The caenorhabditis elegans innexin INX-3 is localized to gap junctions and is essential for embryonic development. Dev. Biol. 256, 403-417. doi: 10.1016/S00121606(02)00116-1

Starich, T. A., Xu, J., Skerrett, I. M., Nicholson, B. J., and Shaw, J. E. (2009). Interactions between innexins UNC-7 and UNC-9 mediate electrical synapse specificity in the Caenorhabditis elegans locomotory nervous system. Neural Dev. 4, 16. doi: 10.1186/1749-8104-4-16

Vinken, M., Decrock, E., Leybaert, L., Bultynck, G., Himpens, B., Vanhaecke, T., et al. (2012). Non-channel functions of connexins in cell growth and cell death. Biochim. Biophys. Acta 1818, 2002-2008. doi: 10.1016/j.bbamem.2011. 06.011

White, J. G., Southgate, E., Thomson, J. N., and Brenner, S. (1986). The structure of the nervous system of the nematode Caenorhabditis elegans. Philos. Trans. $R$ Soc. B. Biol. Sci. 314, 1-340. doi: 10.1098/rstb.1986.0056

Whitten, S. J., and Miller, M. A. (2007). The role of gap junctions in Caenorhabditis elegans oocyte maturation and fertilization. Dev. Biol. 301, 432-446. doi: 10.1016/j.ydbio.2006.08.038

Conflict of Interest Statement: The authors declare that the research was conducted in the absence of any commercial or financial relationships that could be construed as a potential conflict of interest.

Received: 01 November 2013; accepted: 20 January 2014; published online: 11 February 2014.

Citation: Simonsen KT, Moerman DG and Naus CC (2014) Gap junctions in C. elegans. Front. Physiol. 5:40. doi: 10.3389/fphys.2014.00040

This article was submitted to Membrane Physiology and Membrane Biophysics, a section of the journal Frontiers in Physiology.

Copyright (c) 2014 Simonsen, Moerman and Naus. This is an open-access article distributed under the terms of the Creative Commons Attribution License (CC BY). The use, distribution or reproduction in other forums is permitted, provided the original author(s) or licensor are credited and that the original publication in this journal is cited, in accordance with accepted academic practice. No use, distribution or reproduction is permitted which does not comply with these terms. 